\title{
Erratum to: Effects of the KiVa Anti-bullying Program on Adolescents'Depression, Anxiety, and Perception of Peers
}

\author{
Anne Williford • Aaron Boulton • Brian Noland • \\ Todd D. Little • Antti Kärnä • Christina Salmivalli
}

Published online: 8 September 2011

(C) Springer Science+Business Media, LLC 2011

\section{Erratum to: J Abnorm Child Psychol DOI 10.1007/s10802-011-9551-1}

The original version of this article unfortunately contained errors. The error occurred with the calculation of the effect sizes. The entire denominator of the formula should be under a square root sign as written below:

Latent $d=\frac{\alpha_{I}-\alpha_{C}}{\sqrt{\left(\frac{n_{I} \psi_{I}+n_{C} \psi_{C}}{n_{I}+n_{C}}\right)}}$

The online version of the original article can be found at http://dx.doi. org/10.1007/s10802-011-9551-1.

\author{
A. Williford $(\bowtie)$ \\ School of Social Welfare, University of Kansas, \\ 1545 Lilac Lane, \\ Lawrence, KS 66044, USA \\ e-mail: awilliford@ku.edu
}

\author{
A. Boulton · T. D. Little \\ Department of Quantitative Psychology, University of Kansas, \\ Lawrence, KS, USA \\ B. Noland \\ Department of Clinical Psychology, University of Kansas, \\ Lawrence, KS, USA
}

\author{
A. Kärnä \\ Department of Behavioural Sciences and Philosophy, \\ University of Turku, \\ Turku, Finland \\ C. Salmivalli \\ Department of Psychology, University of Turku, \\ Turku, Finland \\ C. Salmivalli \\ Faculty of Arts and Education, University of Stavanger, \\ Stavanger, Norway
}

The values found on Table 2 are incorrect please refer to the next page for the corrected Table 2. Due to incorrect values of Table 2, it affects the scientific contents of the Mean Comparisons section under the Results section. To better understand we have rewritten the whole content of Mean Comparisons section and the corrected data are emphasized in bold letters.

\section{Mean Comparisons}

The CFA model used to test for mean differences fit thedata well $(\chi 2(494)=4100.897, p=<0.01$, RMSEA $=$ $0.043(90 \%$ C.I. $=0.042-0.045), \mathrm{CFI}=0.969, \mathrm{TLI}=0.962$, $\mathrm{SRMR}=0.039$ ). Effect sizes for between-condition mean comparisons can be found in Table 2. The intervention and control conditions were statistically equal on peerreported victimization at wave $1(\Delta \times 2(1)=0.19, p=$ 0.66). A small effect size $(\boldsymbol{d}=\mathbf{0 . 0 1})$ indicated that more victimization occurred in the intervention group at baseline. However, students in the intervention condition reported significantly less victimization at wave $2(\Delta \times 2$ $(1)=13.68, p<0.01)$ and wave $3(\Delta \times 2(1)=57.11, p<$ $0.01)$. The effect sizes for these differences were $-\mathbf{0 . 0 9}$ at wave 2 and $\boldsymbol{d}=\mathbf{- 0 . 1 6}$ at wave 3 . This suggests that students in the intervention condition reported less victimization as compared to the control condition over the course of the study.

Somewhat similar patterns were found for the other three outcome variables. Students' positive peer perceptions actually decreased in both conditions, but the decrease was less dramatic in the intervention condition. Specifically, both conditions' peer ratings were 
Table 2 Between condition effects sizes

\begin{tabular}{lccr}
\hline Factor & W1 & W2 & W3 \\
\hline Peer-reported victimization & 0.01 & -0.09 & -0.16 \\
Perception of peers & 0.02 & - & 0.08 \\
Depression & 0.01 & - & -0.04 \\
Anxiety & -0.03 & - & -0.11 \\
\hline
\end{tabular}

W1 Wave 1, W2 Wave 2, W3 Wave 3

equal at wave $1(\boldsymbol{d}=\mathbf{0 . 0 2} ; \Delta \chi 2(1)=0.56, p=0.46)$ but significantly different at wave $3\left(\boldsymbol{d}=\mathbf{0 . 0 8} ; \Delta \chi^{2}(1)=5.50\right.$, $p=0.02)$. Likewise, mean depression levels increased less dramatically for the intervention conditions as compared to the control condition. However, this effect failed to reach statistical significance. The conditions reported equal levels of depression at wave $1(\boldsymbol{d}=\mathbf{0 . 0 1} ; \Delta \chi 2(1)=$ $0.07, p=0.80)$ and at wave $3(\boldsymbol{d}=-\mathbf{0 . 0 4} ; \Delta \chi 2(1)=2.98, p=$ $0.08)$. Finally, anxiety showed decreases in both conditions over time, though a larger decrease was reported for those receiving the intervention. The conditions reported equal levels of anxiety at wave $1(d=-0.03$; $\left.\Delta \chi^{2}(1)=1.27, p=0.26\right)$ but not at wave $3\left(\boldsymbol{d}=-\mathbf{0 . 1 1} ; \Delta \chi^{2}\right.$ $(1)=21.84, p<0.01)$. 